\title{
Influence de la pénicilline sur la technologie et la qualité du fromage Feta fabriqué à partir du lait de brebis
}

\author{
par \\ E. M. Anifantakis*
}

INTRODUCTION

La qualité du lait utilisé joue un rôle primordial sur la qualité des fromages. La présence d'antibiotiques influence le développement des bactéries qui existent dans le lait et celui des ferments lactiques ajoutés comme les Str. thermophilus, Str. lactis, Str. cremoris, les lactobacillus et, par conséquent, la maturation elle-même.

Bradfield et al. [1] ont constaté que la présence de $0,25 \mu \mathrm{g}$ de chlorotetracycline/ml de lait empêche le développement de l'acidité et de l'arôme dans le fromage Cheddar, tandis qu'elle favorise le développement d'un goût amer. Thomas et Panes [8] ont signalé que les cultures acidifiantes utilisées ne se développent pas du tout quand le lait contenait 0,2 u.i. de pénicilline par ml. Cette action était nette à 0,05 u.i. et faible à $0,01-0,04$ u.i./ml.

Kosikowski et Mocquot [5] ont observé que les fromages qui subissent une fermentation propionique sont influencés défavorablement pendant leur maturation par la présence de $0,01 \mathrm{u}$.i. de pénicilline/ $\mathrm{ml}$ de lait et ils avaient attribué cet effet à la grande sensibilité de Str. thermophilus et Lact. bulgaricus qui jouent un rôle considérable pendant la maturation de ces fromages. Selon Nikolov [6], ces microorganismes présentent des altérations morphologiques quand ils se développent dans le lait qui contient 0,015-0,05 u.i. de pénicilline ou 0,3-0,1 $\mu \mathrm{g}$ d'oxytetracycline, ou 5-7 $\mu \mathrm{g}$ de streptomycine par $\mathrm{ml}$. La pos-

\footnotetext{
* Maître de Conférences, Ecole Supérieure d'Agriculture d'Athènes, Chaire de technologie laitière. Votanikos-Athènes (Grèce).
} 
sibilité d'éliminer les antibiotiques dans le lait, par des méthodes chimiques ou autres, est limitée.

Ce que nous avons noté pour les antibiotiques et leur action dans le lait, se rapporte au lait de vache, qui présente beaucoup de différences par rapport au lait de brebis quant à la composition et aux caractères physico-chimiques. En dehors de cela, le lait de brebis constitue un meilleur substrat pour le développement des cultures lactiques, et il nécessite une manipulation différente de celle du lait de vache pendant la fabrication des fromages. Il est possible que l'action des antibiotiques dans le lait de brebis ne s'identifie pas à celle qui a été observée dans le lait de vache.

Dans la présente étude nous examinons l'action de la pénicilline sur la qualité du fromage Feta fabriqué à partir de lait de brebis, et nous signalons la limite de concentration de la pénicilline au-delà de laquelle le lait de brebis est jugé impropre à la fabrication. En même temps, nous avons examiné l'action d'autres antibiotiques sur le temps de prise et sur l'évolution de la fermeté du gel.

\section{MATERIEL ET METHODES}

Le lait de brebis qui a été utilisé provenait d'un troupeau de 85 brebis de l'Ecole Supérieure d'Agronomie d'Athènes, qui comprend des brebis de race Chios, de Zaanen et de leurs croisements. C'était un mélange de la traite du matin et de l'après-midi, sans antibiotique, avec un poids spécifique de 1,036-1,038 et une teneur en matière grasse de 5,9-6,9 p. 100. Le lait de vache provenait également de l'étable de l'Ecole. Comme levain lactique, nous avons utilisé les souches de ferments $n^{\circ} 25 / 30$ de la maison Equilait qui se composent de microorganismes Str. lactis et Str. cremoris.

Pour la mesure du temps de prise, nous avons adopté la méthode Scott Blair et Burnett [7]. L'humidité du fromage a été déterminée par séchage de $5 \mathrm{~g}$ jusqu'à poids constant à $100^{\circ} \pm 2^{\circ} \mathrm{C}$ et le $\mathrm{pH}$ avec un $\mathrm{pH}$ mètre Radiometer Copenhagen. Nous avons utilisé comme antibiotiques la pénicilline, l'oxytétracycline et la chlorotétracycline fournies par U.S.P. (Références Standards). Pour chaque essai et pour chacun des antibiotiques nous avons fait cinq répétitions. Pour la fabrication des fromages, le lait était pasteurisé à $63^{\circ} \mathrm{C}$ pendant $30 \mathrm{~min}$ et refroidi à $35^{\circ} \mathrm{C}$; nous avons ajouté 1 p. 100 de levain lactique et ensuite nous avons réparti en quantités égales, dans quatre cuves, dont trois contenaient la quantité nécessaire d'antibiotique, de façon que, avec la quantité de lait ajoutée nous obtenions les concentrations voulues de pénicilline. Dans la quatrième cuve, nous n'avons pas mis d'antibiotique, afin d'obtenir le fromage pris comme témoin. La technologie que nous avons suivie est celle décrite par Veinoglou et al. [9]. 


\section{RESULTATS ET DISCUSSION}

Au tableau 1 nous donnons les résultats rapportant l'action des antibiotiques pénicilline, chlorotétracycline et oxytétracycline sur le temps de prise du lait. Comme temps de prise, nous prenons la période de temps à partir de l'addition de la présure jusqu'à l'apparition des premiers agrégats visibles de caséine.

D'après les éléments de ce tableau nous pouvons constater que l'addition de ces antibiotiques aux concentrations étudiées n'a pas d'influence importante sur le temps de prise du lait de brebis qui, de ce point de vue, paraît normal. Nous avons aussi examiné, avec des critères empiriques, à l'aide du torsiomètre Scott-Blair, l'action des antibiotiques sur l'évolution de la fermeté du caillé jusqu'à son découpage. Il est reconnu que cela représente un stade important de la fabrication.

\section{$T A B L E A U 1$}

Action des antibiotiques : pénicilline, chlorotétracycline et oxytétracycline sur le temps de floculation du lait de brebis

\begin{tabular}{|c|c|c|c|c|}
\hline $\begin{array}{c}\text { Sorte } \\
\text { d'antibiotique }\end{array}$ & $\begin{array}{c}\text { Quantité } \\
\text { d'antibiotique } \\
\text { par ml de lait }\end{array}$ & $\begin{array}{l}\text { Temps de } \\
\text { floculation } \\
\text { (en sec) }\end{array}$ & $\begin{array}{l}\text { Différence } \\
\text { par rapport } \\
\text { au témoin }\end{array}$ & $\begin{array}{c}\mathrm{t} \text { (Dunnet) } \\
\mathrm{x} \mathrm{Sd})=\mathrm{d}(3)\end{array}$ \\
\hline Témoin & 0,0 & 523 & - & \\
\hline $\begin{array}{l}\text { Pénicilline } \\
»\end{array}$ & $\begin{array}{r}0,2 \text { u.i. } \\
20,0 \text { u.i. }\end{array}$ & $\begin{array}{l}515 \\
515\end{array}$ & $\begin{array}{l}+8 \\
+8\end{array}$ & \\
\hline Chlorotétracycline & $\begin{array}{r}10,0 \mu \mathrm{g} \\
100,0 \mu \mathrm{g}\end{array}$ & $\begin{array}{l}526 \\
524\end{array}$ & $\begin{array}{l}-3 \\
-1\end{array}$ & $\pm 14,73$ \\
\hline Oxytétracycline & $\begin{array}{r}10,0 \mu \mathrm{g} \\
100,0 \mu \mathrm{g}\end{array}$ & $\begin{array}{l}520 \\
518\end{array}$ & $\begin{array}{l}+3 \\
+3\end{array}$ & \\
\hline
\end{tabular}

Nous avons conclu que ces antibiotiques, dans les conditions de réalisations de nos essais, n'avaient aucune action sur le raffermissement du gel et n'imposaient pas de modification du temps de découpage du caillé durant la fabrication du Feta. Il faut noter que les antibiotiques ont été ajoutés au lait peu après l'addition des ferments lactiques et la présure peu après l'addition des antibiotiques. Par conséquent, il n'y avait pas assez de temps pour que la microflore du lait sans antibiotique lui occasionne des altérations qui le différen- 
cieraient du lait avec antibiotiques et qui se refléteraient sur les qualités du caillé produit. Il est évident que les résultats seraient sans doute différents dans le cas de fromages pour lesquels le temps de maturation du lait, et le temps de coagulation, sont longs.

Le $\mathrm{pH}$ et l'humidité des fromages fabriqués avec du lait de brebis sans antibiotiques ou avec $(0,1-0,2-0,4$ u.i. de pénicilline $\mathrm{G} / \mathrm{ml})$ étaient déterminés le lendemain de la fabrication et 90 jours plus tard. Les résultats sont donnés au tableau 2.

\section{TABLEAU 2}

Humidité et $\mathrm{pH}$ du fromage Feta fait de lait de brebis sans ou avec pénicilline, 1 et $90 \mathrm{j}$ après sa fabrication

\begin{tabular}{|c|c|c|c|c|}
\hline \multirow{3}{*}{$\begin{array}{l}\text { Quantité } \\
\text { de pénicilline } \\
\text { u.i. } / \mathrm{ml} \text { de lait }\end{array}$} & \multicolumn{4}{|c|}{ Fromage Feta } \\
\hline & \multicolumn{2}{|c|}{$1 \mathrm{j}$} & \multicolumn{2}{|c|}{$90 \mathrm{j}$} \\
\hline & Humidité & $\mathrm{pH}$ & Humidité & $\mathrm{pH}$ \\
\hline 0,000 & 60,7 & 5,05 & 52,6 & 4,41 \\
\hline 0,100 & 60,5 & 5,29 & 52,7 & 4,48 \\
\hline 0,200 & 61,8 & 5,62 & 54,7 & 4,65 \\
\hline 0,400 & 61,8 & 5,85 & 54,9 & 4,61 \\
\hline D.M.S. pour $\mathrm{p}=0,5$ & $\pm 0,5$ & $\pm 0,29$ & $\pm 0,9$ & $\pm 0,10$ \\
\hline $\mathrm{p}=0,01$ & $\pm 0,8$ & $\pm 0,40$ & $\pm 1,0$ & $\pm 0,14$ \\
\hline
\end{tabular}

D.M.S. = Différence significative statistiquement.

Nous notons que nous n'avons pas utilisé de concentrations de pénicilline inférieures à $0,1 \mathrm{u}$.i./ml parce que, dans nos essais préliminaires avec $0,05 \mathrm{u}$.i./ $/ \mathrm{ml}$, nous n'avons pas constaté d'action défavorable sur la qualité du Feta.

D'après les éléments de ce tableau nous concluons que l'addition de 0,100 u.i. de pénicilline/ml de lait semble avoir une légère action sur le $\mathrm{pH}$ des fromages âgés de 1 et $90 \mathrm{j}$, mais la différence observée ne peut pas être attribuée avec certitude à l'antibiotique. 
En revanche, avec l'addition de 0,2 u.i./ml ou 0,4 u.i. $/ \mathrm{ml}$ de lait, l'humidité ainsi que le $\mathrm{pH}$ des fromages présentent des différences significatives, par rapport au témoin pour les seuils de probabilité $\mathrm{p}=0,05$ et $\mathrm{p}=0,01$. Les fromages à $90 \mathrm{j}$ avaient aussi un taux d'humidité et un $\mathrm{pH}$ plus élevés.

Ces augmentations de l'humidité et du $\mathrm{pH}$ des fromages sont le résultat de l'action défavorable de l'antibiotique sur leur microflore acidifiante. Elles ne sont pas sans incidence puisque le $\mathrm{pH}$ et le taux d'humidité, pendant les divers stades de la maturation, sont les facteurs les plus importants pour le contrôle de la qualité du produit final [2].

Thomas et Panes [8] ont étudié la fabrication de différents fromages avec du lait de vache et ont constaté que la présence de 0,040,15 u.i. de pénicilline $/ \mathrm{ml}$ de lait donnait des fromages d'une qualité inférieure à celle des témoins avec une acidité anormale, une humidité élevée, une texture spongieuse due aux coliformes et, parfois, un goût amer ou doux.

Emmons et Beckett [4] ont constaté qu'un pH élevé lors du découpage du caillé et aux stades suivants de la fabrication du fromage a une nette influence sur son humidité et sa qualité.

Sur les fromages, nous avons pratiqué également des examens organoleptiques après 2 mois (temps de maturation du fromage Feta), avec cinq dégustateurs, pour déterminer l'influence de la pénicilline sur leur qualité, avec les résultats suivants :

a) La présence de $0,1 \mathrm{u}$.i. de pénicilline/ml de lait n'a pratiquement pas d'action sur la qualité des fromages qui ont presque toujours une bonne texture et un bon goût. Mais il y a eu un cas où cette addition a détérioré la qualité des fromages. Ce fait peut être attribué à ce que la présence de l'antibiotique a facilité la prédominance d'une microflore indésirable. De ce point de vue, la qualité de la microflore du lait lui-même joue un rôle important.

b) La présence de 0,2 ou 0,4 u.i. de pénicilline $/ \mathrm{ml}$ de lait a conduit à la fabrication de fromages de moyenne ou de mauvaise qualité. Ils présentaient un grand nombre de trous irréguliers dans leur masse, leur odeur n'était pas agréable, leur goût était moyen ou mauvais et leur pâte se brisait facilement. La figure 1 nous montre une coupe de fromage Feta âgé de $1 \mathrm{j}$ où l'on peut voir certains défauts caractéristiques.

En conclusion, nous estimons que les concentrations élevées des antibiotiques étudiés n'ont pas une influence appréciable sur le temps de prise et l'évolution de la fermeté du caillé pendant la fabrication du Feta avec du lait de brebis. En revanche des concentrations plus faibles de pénicilline $(0,2$ à 0,4 u.i./ml) ont une action défavorable sur la qualité de ce fromage. Dès le lendemain de la fabrication, nous avons trouvé des différences de $\mathrm{pH}$, d'humidité et de texture. La concentration de 0,1 u.i. de pénicilline/ml n'a pas eu d'action sensible sur la 


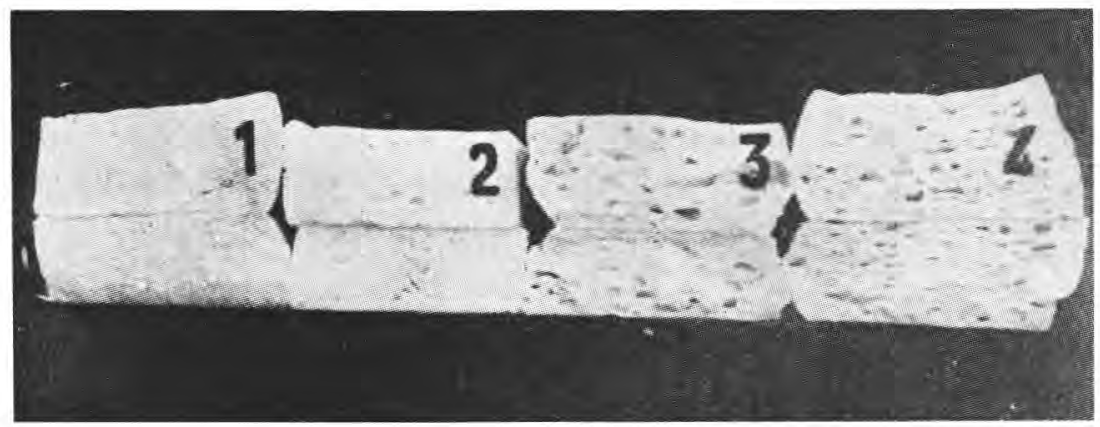

fig. 1

qualité mais il faut rappeler que la qualité bactériologique du lait joue dans ce cas un rôle important.

Les concentrations de pénicilline inférieures à $0,1 \mathrm{u}$.i./ml, utilisées dans les essais préliminaires, n'influencent pas nettement la qualité des fromages. Nous pouvons, par conséquent, dire que le lait de brebis qui contient plus de 0,1 u.i. de pénicilline $/ \mathrm{ml}$ ne doit pas être utilisé pour la fabrication du fromage Feta.

\section{Remerciements}

Nous remercions vivement M. le $\operatorname{Pr}$ B. Veinoglou pour ses précieux conseils. Nous tenons à remercier aussi M. G. Kalatjopoulos de ses conseils pour la rédaction de ce travail.

\section{Rés u mé}

Dans une série d'essais nous avons étudié l'influence de différentes concentrations de pénicilline sur le temps de prise du lait de brebis et l'évolution de la fermeté du caillé durant la fabrication du fromage Feta. Nous avons constaté que ces caractères ne sont pas influencés considérablement. Il en est de même avec les antibiotiques oxytétracycline et chlorotétracycline. Nous avons examiné aussi l'action de la pénicilline à $0,1-0,2$ et 0,4 u.i./ml, de lait de brebis sur la qualité du Feta, et nous avons trouvé que la concentration $0,1 \mathrm{u} . \mathrm{i} . / \mathrm{ml}$ influence très peu la qualité, tandis que les concentrations 0,2 et $0,4 \mathrm{u} . \mathrm{i} . / \mathrm{ml}$ étaient défavorables et pouvaient même parfois être à l'origine de défauts très marqués. Les fromages avec 0,2 et $0,4 \mathrm{u}$.i./. $\mathrm{ml}$ de pénicilline $\mathrm{G}$ à l'âge de 1 et $90 \mathrm{j}$ présentaient une augmentation de l'humidité et du $\mathrm{pH}$. Le fromage mûr présentait un grand nombre de trous irréguliers, une odeur désagréable, un goût moyen à mauvais et une texture qui se brisait facilement. La qualité du lait joue un rôle important sur le degré d'intensité de ces défauts. 


\section{S u m m a r y \\ CONCENTRATIONS OF PENICILLIN IN EWE'S MILK ON THE QUALITY OF FETA CHEESE}

The effect was examined of different concentrations of penicillin in ewe's milk on the quality of Feta cheese. It was found that the presence of 0,1 i.u. penicillin $\mathrm{G}$ per $\mathrm{ml}$ of milk does not affect the quality of the produced cheese while 0,2 or 0,4 i.u. penicillin $\mathrm{G} / \mathrm{ml}$ of milk deteriorates the quality of the cheese. In the latter case the cheese produced was of inferior quality with a high number of small and big holes, bad flavour and tended to break to pieces easily.

Reçu pour publication en mars 1980.

\section{Bibliographie}

[1] Bradfield (A.), Resi (L. A.), Johnstone (D. B.) (1952). - The presence of aureomycin in milk and its effects on cheese making and starter activity. J. Dairy Sci., 35, 51.

[2] Davis (J. G.) (1965). - Cheese (book vol. 1, p. 163).

[3] Dunnet (G. W.) (1955). - A multiple comparisons procedure for comparing several treatments with a control. J. Am. St. Assoc., 50, 1096-1121.

[4] Emmons (D. B.), Becketт (D. C.) (1970). - Control of pH during cutting and cooking in manufacturing cottage cheese. Int. Dairy Congr., vol. E, 355.

[5] Kosikowski (F.V.), Mocouot (G.) (1958). - Advances in cheese technology. F.A.O., 958, 21.

[6] Nikolov (N. M.) (1966). - Effect of antibiotics on the microflora of yoghurt. Dairy Sci. Abs., 28, 75.

[7] Scott Blair (G.), Burnett (J.) (1963). - A simple method for detection an early stage in coagulation of rennetted milk. J. Dairy Res., 30, 383.

[8] Thomas (S. H.), Panes (J. J.) (1955). - The effect of penicillin in milk used for the manufacture of Cheshire cheese. J. Soc. of Dairy Techn., 8, 97.

[9] Veinoglou (B. C.), Kalatzopoulos (G. C.), Stamelos (N.), Anifantakis (E. M.) (1969). - Contribution to the study of the technology and composition of Feta cheese made from sheep's milk Greek Agric. Bank Bul., 168, 1. 